\title{
INCIDENCIA DE LAS INSTITUCIONES DE APOYO A LA CREACIÓN DE EMPRESAS EN LA CIUDAD DE SINCELEJO AÑO 2010 y 2011
}

Autores: Santander José De La Ossa Guerra.(1)-Fernando Hernández Taboada (2) .-Jorge Hernández Ruydiaz (3)

\section{RESUMEN}

$\mathrm{E}$ 1 fomento productivo a través de las MIPYMES, así como la promoción del emprendimiento por parte del estado, consolidan un marco institucional de apoyo a la creación de empresas, que facilitan el desarrollo económico local, aprovechando las potencialidades del microentrono. Este trabajo describe la incidencia de los servicios ofrecidos por las entidades de apoyo a la creación de empresas en la ciudad de Sincelejo, determinando el número de unidades productivas, el nivel de inversión y el sector de la economía, entre los años 2010 y 2011. Se realizó un estudio exploratoriodescriptivo, tomando de manera intencional como población objeto la base de proyectos financiados por el fondo emprender que se hubiesen gestionado a través de las unidades de emprendimiento de la Universidad de Sucre, CECAR, la Corporación Incubadora de Empresas de Sucre y el Centro de la Innovación, la Tecnología y los Servicios del Sena regional Sucre. Los resultados mostraron que en la ciudad de Sincelejo se crearon, 8 empresas en el año 2010 y 4 en el año 2011 financiadas por el fondo emprender. El nivel de inversión canalizado fue de $\$ 524.785 .000$ para el año 2010 y de $\$ 323.502 .400$ para el año 2011. El sector de negocios más representativo, donde se crearon estas empresas, fue el sector de agricultura, ganadería, caza y silvicultura. La incidencia en términos relativos se cuantifica con el 0,65\% para el 2010 y el 0,41\% para el 2011, siendo la unidad de emprendimiento del Sena seccional Sucre la de mayor capacidad de gestión .

\section{Pala bras claves: Emprendimiento, Incidencia, MIPYMES}

\section{ABSTRACT}

$\mathrm{P}$ roductive development through MIPYMES, as well as the promotion of entrepreneurship by the state, consolidate an institutional framework to support the creation of companies, which facilitate local economic development, taking advantage of the potential of the microenterprise. This paper describes the incidence of the services offered by the support entities for the creation of companies in the city of Sincelejo, determining the number of productive units, the level of investment and the economy sector, between 2010 and 2011. An exploratory-descriptive study was carried out, intentionally taking as an object population the base of projects financed by the fund to undertake that had been managed through the entrepreneurship units of the University of Sucre, CECAR, the Succor Business Incubator Corporation and the Sucre Regional Center for Innovation, Technology and Services. The results showed that in the city of Sincelejo, 8 companies were created in 2010 and 4 in 2011 financed by the fund to undertake. The level of investment channeled was $\$ 524,785,000$ for 2010 and $\$ 323,502,400$ for 2011 . The most representative business sector, where these companies were created, was the agriculture, livestock, hunting and forestry sector. The incidence in relative terms is quantified with $0.65 \%$ for 2010 and $0.41 \%$ for 2011 , with the entrepreneurial unit of the SENA section of Sucre having the greatest management capacity.

Keywords: Entrepreneurship, Incidence, MIPYMES.

(1)Administrador de Empresas, Especialista en Gerencia de Mercadeo, Magister en Administración, Magister en Educación, Docente de Planta de la Universidad de Sucre. (2) Ingeniero Agroindustrial, Especialista en Gerencia de Mercadeo, Magister en Administración, Docente de Planta Universidad de Sucre. (3) Ingeniero Agroindustrial, Magister en Ciencias Agroalimentaria, Docente de Planta Universidad de Sucre 


\section{INTRODUCCIÓN}

D esde el año de 1990 se percibe en Colombia, un discurso favorable al desarrollo regional, expresado en la constitución política del país y los planes nacionales de desarrollo, lo anterior es coherente con las reformas de política económica aplicadas en América Latina y el Caribe, donde se pasa del paradigma de gestionar el desarrollo económico desde el centro a la periferia, y se piensa en el desarrollo económico como un proceso endógeno, dependiente de la iniciativa local para el aprovechamiento de las potencialidades del micro entorno. De éste discurso, se materializan una serie de estrategias, enfocadas básicamente en los sectores de infraestructura económica básica, ordenamiento territorial y uso del suelo, fomento productivo empresarial, especialmente MIPYMES, desarrollo de cadenas productivas, políticas de turismo, promoción de exportaciones, atracción de inversiones y mejoramiento de la competitividad (Caicedo, 2008).

El fomento productivo a través de la micro, pequeña y mediana empresa, se materializa con la aprobación y promulgación de la ley 590 de 2000 o ley MIPYME, la cual es reformada por la ley 905 de 2004. En términos generales, dentro de los objetivos de la mencionada ley se pretende:

* la creación de la mayor cantidad de micro, pequeñas y medianas empresas.

* inducir el establecimiento de mejores condiciones de entorno institucional para la creación de las mismas.

* $\quad$ propiciar la formación de capital humano, la asistencia para el desarrollo tecnológico y el acceso a los mercados financieros institucionales.

* $\quad$ promover la permanente formulación, ejecu- ción y evaluación de políticas públicas favorables al desarrollo y a la competitividad de dichas empresas.

La ley MIPYME, se configura en una herramienta de política pública, que establece un marco institucional, con el objeto de coadyuvar la creación del mayor número de micro, pequeñas y medianas empresas a través de la alianza entre el sector público, privado y sin ánimo de lucro, así mismo reconoce que es necesario promover la permanente formulación y ejecución de políticas públicas favorables a la creación de empresas.

Consecuente con la ley MIPYME, el estado promulga la ley 1014 de 2006, conocida como "ley de fomento de cultura al emprendimiento" como objeto central de la ley de emprendimiento, entre otros, se tiene:

* la promoción del espíritu emprendedor a través de los estamentos educativos del país (el emprendimiento, como cultura, se inserta en el currículo),

* $\quad$ la creación de vínculos entre el sistema productivo y el sistema educativo.

* el fortalecimiento del marco institucional de apoyo a la creación de empresas.

Con la ley de emprendimiento, el estado busca promover el espíritu emprendedor, a través del sistema educativo, haciéndolo parte del currículo de formación, por lo que todos los colegios quedan obligados a incluir en sus proyectos educativos institucionales la cátedra de emprendimiento, en el mismo sentido busca establecer vínculos entre el sistema educativo y el sistema productivo y por supuesto, centra su atención, al igual que la ley MIPYME, en consolidar un marco institucional de apoyo a la creación de empresas. 
La ley MYPIME y la ley de Emprendimiento, configuran un escenario de oportunidades para la creación de empresas, de igual forma con la implementación de la ley, se ha desarrollado, toda una oferta de servicios de poyo, brindados por empresas estatales, sin ánimo de lucro, organizaciones no gubernamentales, empresas privadas; las cuales, están representadas a nivel nacional, regional y local en: Incubadoras, Centro de Desarrollo del Espíritu Empresarial, Centros de Desarrollo Empresarial, Centros de Competitividad, Centros de Desarrollo Productivo y Entidades que desarrollan capacitación en temas de Creación de Negocios (Varela, 2008).

En ese sentido es pertinente preguntarse: ¿Cuál fue la incidencia de las entidades de apoyo a la creación de empresas en la ciudad de Sincelejo en los años 2010 y 2011 ?

Se pretende, por lo tanto, en el presente trabajo describir la incidencia de las entidades de apoyo a la creación de empresas en la ciudad de Sincelejo, buscando determinar: el número de empresas creadas, con la ayuda de las entidades de apoyo; el nivel de inversión que se canalizó para el sector empresarial de la ciudad producto de convocatorias de entidades de apoyo y el sector de negocio, donde se ha creado el mayor número de empresas, producto de los servicios ofrecidos por dichas entidades; en los años antes referenciados. 


\section{Marco Teórico}

\section{Estudio del Concepto de Incidencia}

$\mathrm{L}$ a real academia de la lengua tiene dos definiciones del concepto "incidencia", que satisfacen lo que se pretende hacer con el presente proyecto de investigación, en un primer momento lo define como "número de casos ocurridos" y en un segundo momento lo define como "Influencia o repercusión”. En ese sentido, lo que se pretende mostrar con esta investigación, es la influencia o repercusión que tienen las entidades de apoyo en la creación de empresas en la ciudad de Sincelejo, por lo tanto al momento de medir la incidencia, va a ser necesario determinar el número de casos o el número de empresas que se crean producto del accionar de las entidades de apoyo.

En esta línea de investigación, se enmarca el trabajo realizado por Liyis Gómez de la división de Ciencias Económicas de la Universidad del Norte, en el cual analiza los mecanismos de apoyo a la creación de empresas en el Caribe colombiano. Con el trabajo se pretende mostrar las medidas de apoyo y su incidencia en la oferta y demanda de servicios referentes a la creación de empresas. Por el lado de la demanda analiza el conocimiento, la utilización y la valoración que tiene la población empresarial de dichas medidas, y por el lado de la oferta, mide la capacidad que tienen los organismos de apoyo, para desarrollar los instrumentos que implican las medidas. El trabajo tiene como marco teórico "la teoría económica institucional”, su metodología implica un estudio exploratorio - descriptivo, donde se recaban datos de fuentes primaria y secundaria e involucra dos poblaciones: por un lado los representantes de instituciones de apoyo y por el otro una muestra de empresarios de la región. Los resultados de la investigación identificaron los siguientes aspectos: 1. -que hay poca incidencia de los organismos de apoyo internacional en la generación de empresas de acumulación, los recursos de los orga- nismos de apoyo internacional, están dirigidos a la creación de empresas en población desplazada, orientadas básicamente a la generación de autoempleo. 2. -hay poca presencia de los organismos nacionales en la región Caribe, con excepción del SENA y las entidades financieras. 3. -hay un alto desconocimiento y de no utilización, en la región, de los programas y organismos de apoyo a la creación de empresas; al respecto, los representantes de entidades son conscientes que el tema ha tenido poca promoción (Gómez, 2004).

Un segundo trabajo que se puede resaltar como referente de esta investigación, es el trabajo realizado por Jesus Pájaro García, el cual tuvo como objetivo evaluar el impacto de las políticas de apoyo a la creación de empresas en Cartagena de Indias, destacando la legislación nacional y local aplicada por las instituciones locales y sus programas de apoyo. Al igual que la investigación de Liyis Gómez, la investigación de pájaro se hizo teniendo en cuenta dos poblaciones, por un lado, los representantes de las entidades de apoyo a nivel nacional y local, y por el otro las personas que crearon empresas entra 2000 y 2005, el propósito de la investigación fue identificar qué nivel de empresarios, crearon sus empresas por efecto de las medidas e instituciones de apoyo y cuál era el nivel de conocimiento de las mismas. Entre las conclusiones obtenidas en el estudio se resalta el bajo conocimiento que tienen los empresarios de las medidas de apoyo y el alto porcentaje de empresas creadas en la ciudad, que nada tienen que ver con las políticas estatales de creación de empresas (Pájaro, 2007). 
Marco institucional de apoyo a la creación de empresas en Colombia.

Gráfico N 1. Instituciones de apoyo a la creación de empresas en Colombia

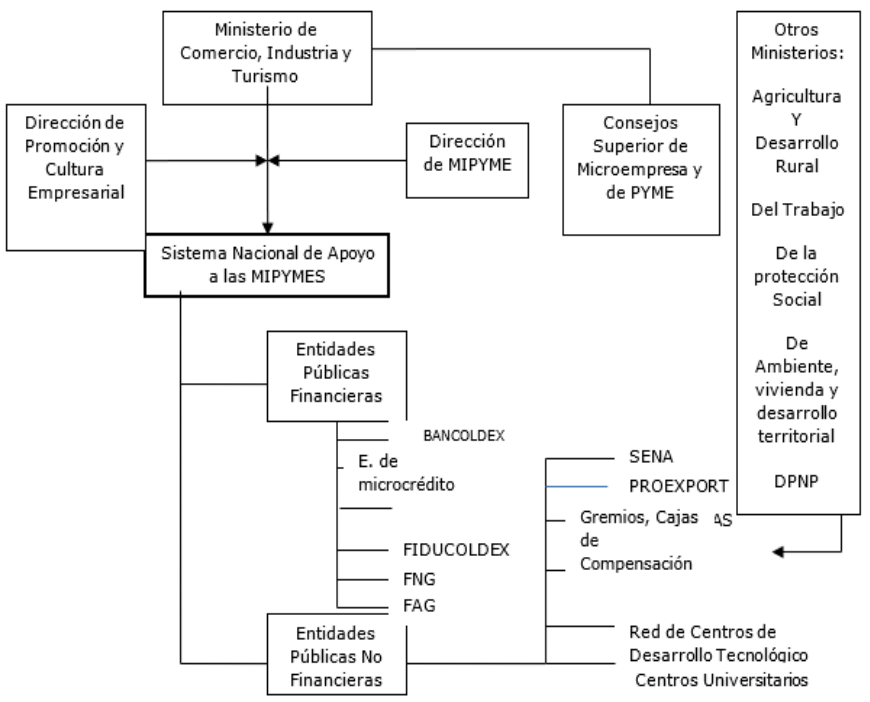

Fuente: Tomado de: Medidas de apoyo a la creación de empresas en el caribe colombiano: "análisis de la oferta y demanda de servicios" Liyis Gómez. Ampliado con análisis propio

En el gráfico No. 1 se relacionan todas las entidades que hacen parte del marco institucional de apoyo a la creación de empresas a nivel nacional, este marco tiene su origen en la ley 590 de 2000 (ley MIPY$\mathrm{ME}$ ), el cual en su capítulo II, introduce como marco institucional "el Sistema Nacional de Apoyo a las Mipymes", integrado por las siguientes instituciones: los ministerios de Comercio, Industria y Turismo, Agricultura y Desarrollo Rural, Del Trabajo, De la protección Social y De Ambiente, Vivienda y Desarrollo Territorial, así mismo, el Departamento Nacional de Planeación, el Sena, Colciencias, Bancóldex, Fondo Nacional de Garantías y Finagro. Posteriormente en el año 2006, con la ley de "Fomento a la cultura del emprendimiento", la cual da vida a la "Red nacional para el emprendimiento", se introduce a este marco institucional al Ministerio de Educación Nacional, debido a que la pretensión de esta norma, es introducir la cátedra de emprendimiento en los currículos de las diferentes instituciones educativas, a fin de generar una cultu- ra de emprendimiento a nivel nacional. En el mismo sentido, introduce representantes de las instituciones de educación superior e involucra a gremios tales como "Acopi" y "Fenalco", por su segundo objetivo macro que consiste en acercar al sector productivo con la academia. De la misma forma se introducen las instituciones de microcrédito, las cajas de compensación familiar y las incubadoras de empresas.

\section{Marco institucional de apoyo a la creación de empresas en Sucre}

Tabla No. 3. Instituciones de apoyo a la creación de empresa en Sucre y Sincelejo.

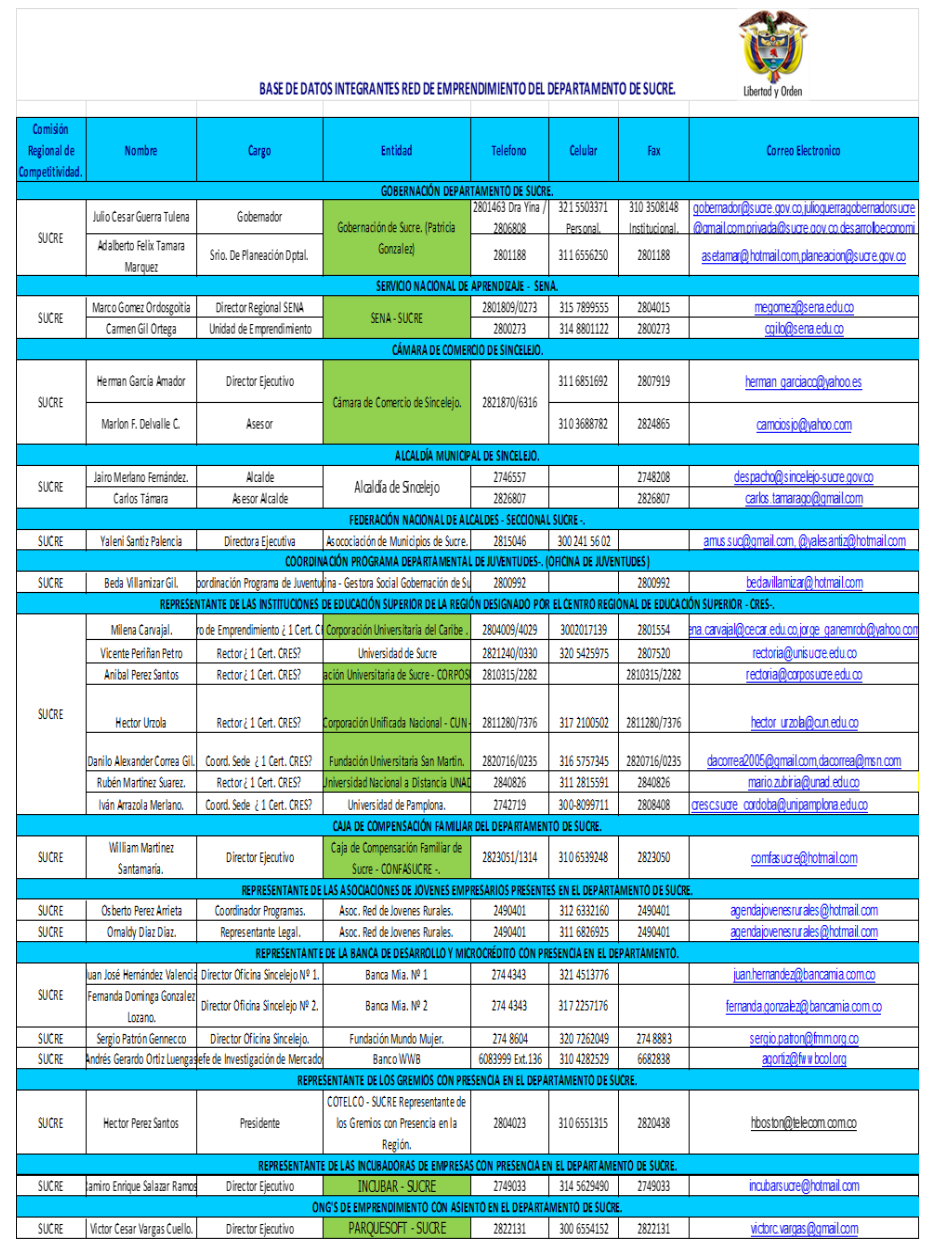

Fuente: Cámara de Comercio de Sincelejo. 
De igual forma que en el ámbito nacional se configura un andamiaje institucional de apoyo a la creación de empresas, en el ámbito departamental hay un cuerpo institucional que encadenado con el sistema de apoyo nacional, facilita los procesos de creación de empresas, a través del acompañamiento y orientación a emprendedores, este marco institucional del Departamento de Sucre, está organizado de acuerdo a lo establecido en la ley de fomento a la cultura de emprendimiento, articulo 6 en lo referente a la "Red Regional de Emprendimiento".

\section{Fondo Emprender y Unidades de Em- prendimiento.}

En la página web del fondo emprender se puede leer la siguiente definición que explica la filosofía del fondo emprender:

"Es un Fondo creado por el Gobierno Nacional para financiar iniciativas empresariales que provengan y sean desarrolladas por alumnos del SENA que hayan finalizado la etapa lectiva de un programa de formación, alumnos o egresados de cursos del SENA en el programa jóvenes rurales y línea de formación de líderes del desarrollo, estudiantes que se encuentren cursando los dos (2) últimos semestres en un programa de educación superior reconocido por el Estado de conformidad con las Leyes 30 de 1992 y 115 de 1994, personas que hayan concluido materias dentro de los últimos doce (12) meses, profesionales universitarios cuyo primer título haya sido obtenido durante los últimos 24 meses, estudiantes o egresados que se encuentren cursando especialización y/o maestría que hayan culminado y obtenido la certificación dentro de los últimos 12 meses. El Fondo Emprender se rige por el derecho privado, y su presupuesto está conformado por el 80\% de la monetización de la cuota de aprendizaje de que trata el artículo 34 de la ley 789 del 2002, así como por los aportes del presupuesto general de la nación, recursos financieros de organismos de cooperación nacional e internacional, re- cursos financieros de la banca multilateral, recursos financieros de organismos internacionales, recursos financieros de fondos de pensiones y cesantías y recursos de fondos de inversión públicos y privados".

La definición del fondo delimita un segmento poblacional de beneficiarios del mismo, que están ligados a las instituciones de educación, es decir, la filosofía del fondo es servir como fuente de financiamiento para la creación de empresas de estudiantes o recién graduados de las instituciones de educación superior.

Las unidades de emprendimiento son dependencias del Sena, Universidades u ONG, que se dedican de manera profesional a la atención de emprendedores y empresarios con el objeto de ofrecer asesorías en todos los procesos propios de la dinámica empresarial.

Tabla No. 4. Servicios que ofrecen las unidades de emprendimiento

\begin{tabular}{|l|l|l|}
\hline \multicolumn{1}{|c|}{ Servicios } & \multicolumn{1}{|c|}{ Procesos } & Público objetivo \\
\hline $\begin{array}{l}\text { Sensibilización } \\
\text { emprendimiento en }\end{array}$ & $\begin{array}{l}\text { Conferencias, foros, } \\
\text { seminarios y charlas }\end{array}$ & Todos los emprendedores \\
\hline $\begin{array}{l}\text { Identificación de ideas de } \\
\text { negocios }\end{array}$ & $\begin{array}{l}\text { Identificación perfil del } \\
\text { emprendedor } \\
\text { identificación de ideas de } \\
\text { negocios }\end{array}$ & Todos los emprendedores \\
\hline $\begin{array}{l}\text { Asesoramiento en fuentes } \\
\text { de financiación }\end{array}$ & $\begin{array}{l}\text { Plan fondo emprender } \\
\text { Otras fuentes de } \\
\text { financiamiento }\end{array}$ & $\begin{array}{l}\text { Los emprendedores que } \\
\text { queden en el proceso }\end{array}$ \\
\hline $\begin{array}{l}\text { Asesoramiento puesto en } \\
\text { marcha }\end{array}$ & $\begin{array}{l}\text { Acompañamientor al } \\
\text { emprendedor hasta poner } \\
\text { en funcionamientor la } \\
\text { empresa }\end{array}$ & $\begin{array}{l}\text { Los emprendedores que } \\
\text { queden en el proceso }\end{array}$ \\
\hline $\begin{array}{l}\text { Fortalecimiento de } \\
\text { empresas }\end{array}$ & $\begin{array}{l}\text { A las empresas constituidas } \\
\text { en el proceso }\end{array}$ & $\begin{array}{l}\text { Los emprendedores que } \\
\text { consolidan el negocio }\end{array}$ \\
\hline Asesorías empresariales & $\begin{array}{l}\text { Emprendimiento, plan de } \\
\text { negocios, mercadeo, } \\
\text { finanzas. }\end{array}$ & $\begin{array}{l}\text { Empresarios que lo } \\
\text { requieran. }\end{array}$ \\
\hline
\end{tabular}

Fuente: http://www.fondoemprender.com/BancoConocimiento/F Fondo Emprender/Fondo Emprender.ASP

En Sincelejo, se encuentran registradas ante el fondo emprender las siguientes unidades de emprendimiento: 
Tabla No. 5 Unidades de emprendimiento con sede en Sincelejo

\begin{tabular}{|l|c|c|}
\hline \multicolumn{1}{|c|}{ INSTITUCIÓN } & CIUDAD & DEPARTAMENTO \\
\hline Universidad de Sucre (UNISUCRE) & Sincelejo & Sucre \\
\hline $\begin{array}{l}\text { Centro de la Innovación, la Tecnología y los } \\
\text { Servicios (SENA - Sucre) }\end{array}$ & Sincelejo & Sucre \\
\hline $\begin{array}{l}\text { Corporación Incubadora de Empresas de Sucre } \\
\text { (Incubar Sucre) }\end{array}$ & Sincelejo & Sucre \\
\hline Corporación Universitaria del Caribe (CECAR) & Sincelejo & Sucre \\
\hline
\end{tabular}

Fuente: http://www.fondoemprender.com/BancoConocimiento/F/ Fondo_Emprender/Fondo_Emprender.ASP

\section{Metodología}

Se hizo un estudio Exploratorio -Descriptivo, con el objeto de mostrar la incidencia que han tenido las entidades de apoyo de creación de empresas en la ciudad de Sincelejo. De manera intencional se toma como población objeto de análisis la base de proyectos financiados por el fondo emprender en los años 2010 y 2011, a través de sus convocatorias, las cuales se encuentran disponibles en el portal web de dicho fondo.

Para organizar, clasificar y dar sentido a la información Se hizo uso del software Excel, a través del cual se construyeron tablas y gráficos, a fin de satisfacer los vacíos de conocimiento que motivaron el proyecto.

La información se clasifica teniendo en cuenta los objetivos de investigación, a través de los cuales se pretende, determinar la incidencia que han tenido las entidades de apoyo de creación de empresas, en el sector empresarial de la ciudad de Sincelejo, para ello es necesario responderse los siguientes cuestionamientos: Cuantas empresas se han creado en Sincelejo, producto de los servicios de las entidades de apoyo?, ¿cuál es el nivel de inversión que se ha canalizado para la ciudad a través de estas entidades? Y ¿cuáles son los sectores de negocios donde más inciden tienen estas iniciativas?

Operativamente lo que se hizo fue revisar la base de proyector del fondo emprender de las convocatorias número $8,9,10,11,12,13$ y 14 , las cuales se desarrollaron entre los años 2010 y 2011; y determinar cuáles de esos proyectos fueron iniciativas de emprendedores de la ciudad de Sincelejo, que se hubiesen gestionado a través de las unidades de emprendimiento de la Universidad de Sucre, la Corporación Educativa del Caribe -CECAR, la Corporación Incubadora de Empresas de Sucre y/o el Centro de la Innovación, la Tecnología y los Servicios del Sena regional Sucre.

\section{Análisis e Interpretación de Resultados}

En el año 2010 el fondo emprender realizó las convocatorias 8,9 y 10 ; con presupuesto de $\$ 10 \mathrm{mil} \mathrm{mi-}$ llones, $\$ 500$ millones y $\$ 15$ mil millones respectivamente, lo cual consolidó un presupuesto total para el año de $\$ 25.500$ millones.

Tabla No. 10 Proyectos por Departamentos convocatorias Fondo emprender- año 2010

\begin{tabular}{|c|c|c|c|}
\hline DEPARTAMENTO & NO VIABLE & VIABLE & $\begin{array}{l}\text { TOTAL, } \\
\text { GENERAI }\end{array}$ \\
\hline Amazonas & 9 & 2 & 11 \\
\hline Antioquia & 39 & 20 & 59 \\
\hline Arauca & 5 & 1 & 6 \\
\hline Archipiélago de San Andrés & 7 & & 7 \\
\hline Atlántico & 43 & 14 & 57 \\
\hline Bogotá D.C & 140 & 29 & 169 \\
\hline Bogotá DC & 9 & & 9 \\
\hline Bolívar & 52 & 9 & 61 \\
\hline Boyacá & 32 & 5 & 37 \\
\hline Caldas & 50 & 12 & 62 \\
\hline Caquetá & 16 & 17 & 33 \\
\hline Casanare & 1 & 2 & 3 \\
\hline Cauca & 64 & 25 & 89 \\
\hline Cesar & 6 & 4 & 10 \\
\hline Chocó & 22 & & 22 \\
\hline Córdoba & 25 & 5 & 30 \\
\hline Cundinamarca & 105 & 21 & 126 \\
\hline Guainía & 1 & & 1 \\
\hline Guaviare & 7 & 4 & 11 \\
\hline Huila & 42 & 32 & 74 \\
\hline La Guajira & 32 & 2 & 34 \\
\hline Magdalena & 9 & 2 & 11 \\
\hline Meta & 15 & 5 & 20 \\
\hline Nariño & 159 & 31 & 190 \\
\hline Norte de Santander & 26 & 12 & 38 \\
\hline Putumayo & 6 & 6 & 12 \\
\hline Quindío & 22 & 9 & 31 \\
\hline Quindío & 5 & & 5 \\
\hline Risaralda & 34 & 15 & 49 \\
\hline Santander & 65 & 27 & 92 \\
\hline Sucre & 59 & 20 & 79 \\
\hline Tolima & 52 & 13 & 65 \\
\hline Valle del Cauca & 87 & 17 & 104 \\
\hline Vichada & 2 & & 2 \\
\hline Total, general & 1248 & 361 & 1609 \\
\hline
\end{tabular}

Fuente: Tomada del Fondo emprender y ajustada polos autores 
En las convocatorias del 2010, se presentaron ante el fondo emprender un total de 1248 proyectos a nivel nacional, de los cuales fueron viabilizados y financiados 361 proyectos. Los emprendedores del departamento de Sucre participaron con 70 proyectos, de los cuales 20 fueron viabilizados y los 50 restantes fueron declarados no viables.

Tabla No.11. Inversión Realizada Convocatorias De 2010

\begin{tabular}{|c|c|c|c|}
\hline DEPARTAMENTO & $\begin{array}{l}\text { PROYECTOS } \\
\text { FINANCIADOS }\end{array}$ & INVERSIÓN & $\begin{array}{c}\% \text { DE } \\
\text { PARTICIPACIÓN }\end{array}$ \\
\hline Amazonas & 2 & 166.860 .000 & 0,70 \\
\hline Antioquia & 20 & 1.382 .775 .000 & 5,82 \\
\hline Arauca & 1 & 67.980 .000 & 0,29 \\
\hline Archipiélago de San Andrés & & - & 0,00 \\
\hline Atlántico & 14 & 1.103 .645 .000 & 4,65 \\
\hline Bogotá D.C & 29 & 2.118 .710 .000 & 8,92 \\
\hline Bogotá DC & & - & 0,00 \\
\hline Bolívar & 9 & 544.870 .000 & 2,29 \\
\hline Boyacá & 5 & 264.710 .000 & 1,11 \\
\hline Caldas & 12 & 821.940 .000 & 3,46 \\
\hline Caquetá & 17 & 1.101 .585 .000 & 4,64 \\
\hline Casanare & 2 & 136.990 .000 & 0,58 \\
\hline Cauca & 25 & 1.568 .690 .000 & 6,60 \\
\hline Cesar & 4 & 214.755 .000 & 0,90 \\
\hline Chocó & & - & 0,00 \\
\hline Córdoba & 5 & 338.870 .000 & 1,43 \\
\hline Cundinamarca & 21 & 1.311 .190 .000 & 5,52 \\
\hline Guainía & & - & 0,00 \\
\hline Guaviare & 4 & 236.385 .000 & 1,00 \\
\hline Huila & 32 & 1.940 .005 .000 & 8,17 \\
\hline La Guajira & 2 & 110.210 .000 & 0,46 \\
\hline Magdalena & 2 & 169.435 .000 & 0,71 \\
\hline Meta & 5 & 281.705 .000 & 1,19 \\
\hline Nariño & 31 & 2.092 .960 .000 & 8,81 \\
\hline Norte de Santander & 12 & 805.460 .000 & 3,39 \\
\hline Putumayo & 6 & 364.105 .000 & 1,53 \\
\hline Quindío & 9 & 648.900 .000 & 2,73 \\
\hline Quindío & & - & 0,00 \\
\hline Risaralda & 15 & 906.400 .000 & 3,82 \\
\hline Santander & 27 & 1.829 .280 .000 & 7,70 \\
\hline Sucre & 20 & 1.338 .485 .000 & 5,64 \\
\hline Tolima & 13 & 812.155 .000 & 3,42 \\
\hline Valle del Cauca & 17 & 1.073 .775 .000 & 4,52 \\
\hline Vichada & & - & 0,00 \\
\hline Total, general & 361 & 23.752 .830 .000 & 100,00 \\
\hline
\end{tabular}

Fuente: Tomada del Fondo emprender y ajustada polos autores

En el año 2010, el fondo emprender concretó una inversión de \$23.752.830.000 a nivel nacional, los emprendedores del departamento de sucre lograron canalizar de esa inversión un 5,64\%, lo que significó 1.338.485.000, para ese año sucre fue el séptimo departamento con el porcentaje de inversión más alto, después del Distrito Capital de Bogotá y los departamentos de Nariño, Huila, Santander, Cauca y Antioquia (ver tabla 11 y gráfico 5).
Gráfico No. 5 Nivel de inversión por departamento convocatorias 2010 en smmly

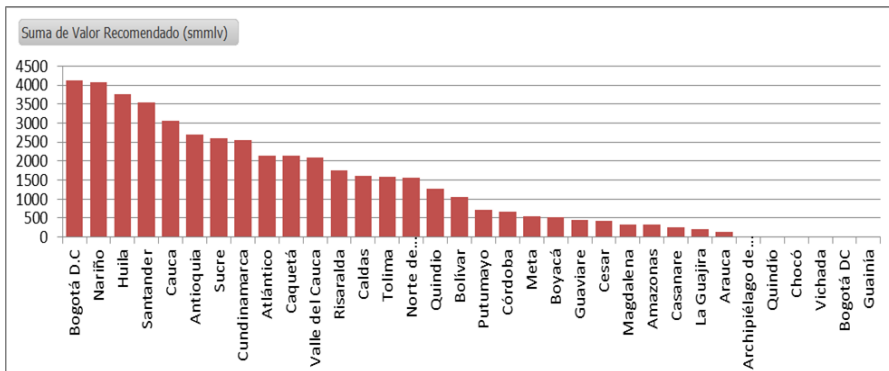

Departamento -

Gráfico No. 6 Proyectos viables en el Departamento de Sucre, clasificados por municipio convocatorias 2010

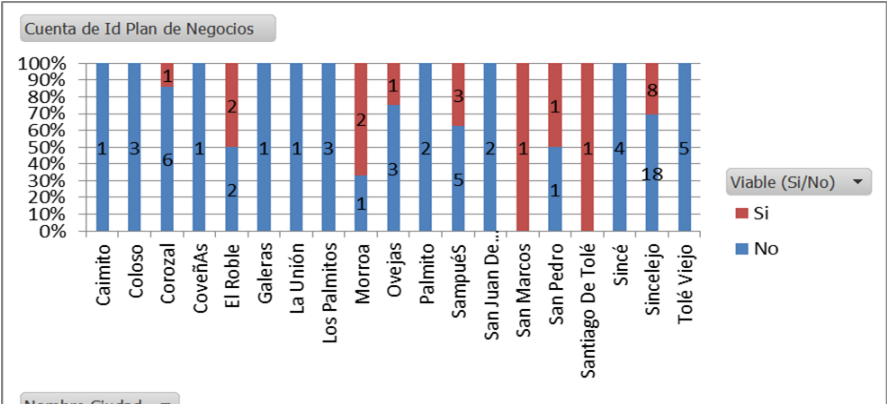

Nombre Giudad -

De los 79 proyectos presentados en Sucre, 26 fueron presentados por emprendedores en Sincelejo, y 53 en el resto del departamento. De los 26 presentados en Sincelejo, 8 fueron viabilizados y 18 fueron declarados como no viables, esto significó, que para la ciudad se lograra un inversión de \$524.785.000,oo, es decir, se logró canalizar para la ciudad el 2,2\% de la inversión nacional y el 39,20 a nivel departamental.

Gráfico No. 7 Proyectos viables en Sincelejo, clasificados por sector convocatorias 2010

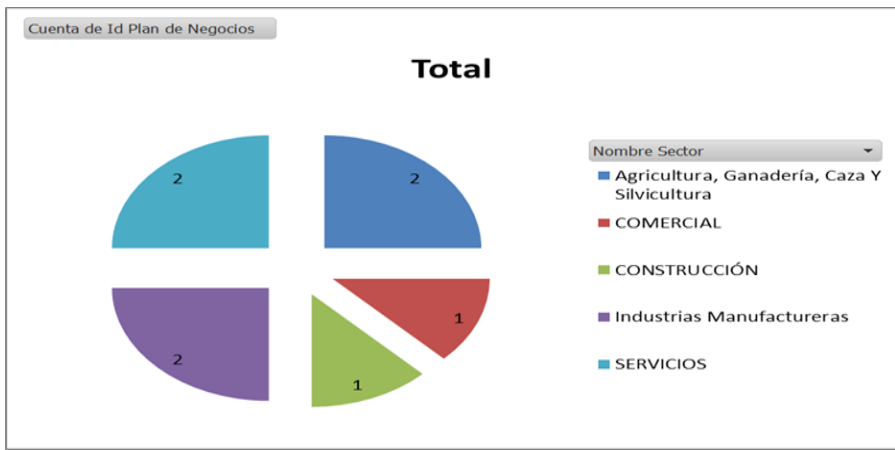


Para el año 2010, los sectores reflejados en los proyectos viabilizados y financiados por el fondo emprender para la ciudad de Sincelejo, fueron en su orden Agricultura, ganadería, Caza y Silvicultura, Comercial, Construcción, Servicios e Industria manufacturera.

En el año 2011, el fondo emprender realizó las convocatorias No.11, 12, 13 y 14, para financiar unidades productivas, con un presupuesto de $\$ 12 \mathrm{mil}$, 5 mil, 3 mil y 10 mil; millones de pesos respectivamente, lo que consolido una inversión total presupuestada de $\$ 30$ mil millones.

Tabla No. 12 Proyectos Por Departamentos Convocatorias Fondo Emprender 2011

\begin{tabular}{|c|c|c|c|}
\hline DEPARTAMENTO & NO VIABLE & VIABLE & $\begin{array}{l}\text { TOTAL, } \\
\text { GENERAL }\end{array}$ \\
\hline Amazonas & 1 & & 1 \\
\hline Antioquia & 72 & 11 & 83 \\
\hline Arauca & 3 & & 3 \\
\hline Archipiélago de San Andrés & 1 & 1 & 2 \\
\hline Atlántico & 45 & 2 & 47 \\
\hline Bogotá D.C & 136 & 14 & 150 \\
\hline Bolívar & 46 & 5 & 51 \\
\hline Boyacá & 15 & 5 & 20 \\
\hline Caldas & 42 & 7 & 49 \\
\hline Caquetá & 13 & 10 & 23 \\
\hline Casanare & 4 & & 4 \\
\hline Cauca & 51 & 7 & 58 \\
\hline Cesar & 5 & 3 & 8 \\
\hline Chocó & 12 & 1 & 13 \\
\hline Córdoba & 41 & 1 & 42 \\
\hline Cundinamarca & 64 & 15 & 79 \\
\hline Guainía & 2 & & 2 \\
\hline Guaviare & 11 & 4 & 15 \\
\hline Huila & 35 & 23 & 58 \\
\hline La Guajira & 10 & 1 & 11 \\
\hline Magdalena & 7 & 1 & 8 \\
\hline Meta & 12 & 2 & 14 \\
\hline Nariño & 158 & 19 & 177 \\
\hline Norte de Santander & 37 & 6 & 43 \\
\hline Putumayo & 7 & & 7 \\
\hline Quindío & 39 & 4 & 43 \\
\hline Risaralda & 30 & 6 & 36 \\
\hline Santander & 78 & 11 & 89 \\
\hline Sucre & 32 & 10 & 42 \\
\hline Tolima & 39 & 10 & 49 \\
\hline Valle del Cauca & 104 & 10 & 114 \\
\hline Vaupés & 2 & & 2 \\
\hline Vichada & 1 & & 1 \\
\hline Total, general & 1155 & 189 & 1344 \\
\hline
\end{tabular}

Fuente:-http://www.fondoemprender.com/BancoConocimiento/C/ convocatoria_nac_no_14/convocatoria_nac_no_14.asp

En las convocatorias de 2011, se presentaron ante el fondo emprender, a través de las unidades de emprendimiento y otras entidades de apoyo, 1155 proyectos, de los cuales fueron viabilizados y financiados 189. Los emprendedores del departamento de Sucre participaron con 42 proyectos, de los cuales fueron viabilizados $\mathrm{y}$ financiados 10 (ver tabla No.12).
Tabla No. 13 Inversión Realizada Convocatorias De 2011

\begin{tabular}{|c|c|c|c|}
\hline DEPARTAMENTO & $\begin{array}{l}\text { PROYECTOS } \\
\text { FINANCIADOS }\end{array}$ & INVERSIÓN & $\begin{array}{c}\% \text { DE } \\
\text { PARTICIPACIÓN }\end{array}$ \\
\hline Amazonas & & - & - \\
\hline Antioquia & 11 & 733.772 .000 & 5,5 \\
\hline Arauca & & - & - \\
\hline Archipiélago de San Andrés & 1 & 93.730 .000 & 0,7 \\
\hline Atlántico & 2 & 176.748 .000 & 1,3 \\
\hline Bogotá D.C & 14 & 1.062 .630 .400 & 7,9 \\
\hline Bolivar & 5 & 347.604 .400 & 2,6 \\
\hline Boyacá & 5 & 317.075 .200 & 2,4 \\
\hline Caldas & 7 & 501.321 .600 & 3,7 \\
\hline Caquetá & 10 & 704.849 .600 & 5,3 \\
\hline Casanare & & - & - \\
\hline Cauca & 7 & 402.771 .200 & 3,0 \\
\hline Cesar & 3 & 156.930 .800 & 1,2 \\
\hline Chocó & 1 & 58.380 .400 & 0,4 \\
\hline Córdoba & 1 & 96.408 .000 & 0,7 \\
\hline Cundinamarca & 15 & 1.052 .989 .600 & 7,9 \\
\hline Guainía & & - & - \\
\hline Guaviare & 4 & 283.332 .400 & 2,1 \\
\hline Huila & 23 & 1.552 .168 .800 & 11,6 \\
\hline La Guajira & 1 & 80.340 .000 & 0,6 \\
\hline Magdalena & 1 & 42.848 .000 & 0,3 \\
\hline Meta & 2 & 176.212 .400 & 1,3 \\
\hline Nariño & 19 & 1.315 .433 .600 & 9,8 \\
\hline Norte de Santander & 6 & 465.972 .000 & 3,5 \\
\hline Putumayo & & - & - \\
\hline Quindío & 4 & 265.122 .000 & 2,0 \\
\hline Risaralda & 6 & 416.161 .200 & 3,1 \\
\hline Santander & 11 & 812.505 .200 & 6,1 \\
\hline Sucre & 10 & 796.437 .200 & 6,0 \\
\hline Tolima & 10 & 727.344 .800 & 5,4 \\
\hline Valle del Cauca & 10 & 730.558 .400 & 5,5 \\
\hline Vaupés & & - & - \\
\hline Vichada & & - & - \\
\hline Total, general & 189 & 13.369 .647 .200 & 100,0 \\
\hline
\end{tabular}

Fuente:-http://www.fondoemprender.com/BancoConocimiento/C/ convocatoria_nac_no_14/convocatoria_nac_no_14.asp

Como resultado de las convocatorias, se financiaron los 189 proyectos viabilizados, con una inversión de \$13.369.647.200, lo que significa que para el año 2011, los emprendedores a nivel nacional sólo se apropiaron del 44,56\% de los recursos presupuestados por el fondo emprender, para financiar sus unidades productivas, el porcentaje de proyectos declarados no viables por los evaluadores del fondo fue del 83,63\%: conocedores del tema, tales como Heraldo Alvis, asesor del fondo emprender, manifiesta que este índice no refleja la calidad de los proyectos, sino más bien la disponibilidad real de los recursos presupuestados que posteriormente obligan al fondo emprender a priorizar los recursos. Con los 10 proyectos viabilizados de emprendedores del departamento de Sucre, se logró una inversión de $\$ 796.437 .200$, lo que significó un 6\% de la inversión total, esa cifra ubicó al departamento en 
el sexto lugar de los departamentos más favorecidos, después del Huila, Nariño, el Distrito capital, Cundinamarca y Santander (ver tabla 9 y gráfico 8)

Gráfico No. 8 Nivel de inversión por departamento convocatorias 2011 en smmlv

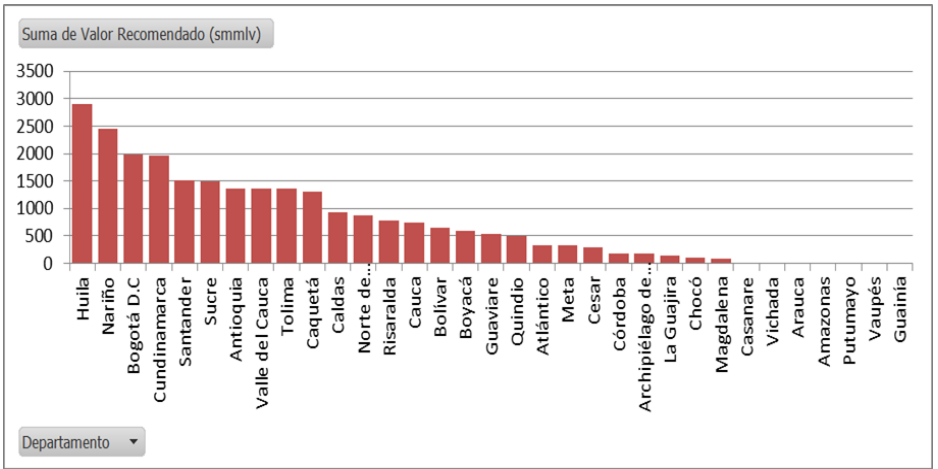

Gráfico No. 9. Proyectos viables en el Departamento de Sucre, clasificados por municipio convocatorias 2011

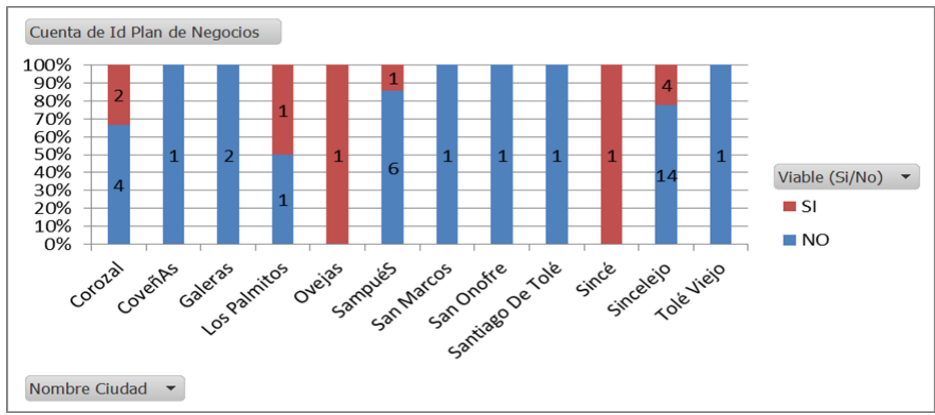

De los proyectos viables del departamento de $\mathrm{Su}-$ cre, 4 tuvieron como ciudad de ejecución, el municipio de Sincelejo, los otros 6, se desarrollaron en municipios como Corozal, Coveñas, Galeras y Palmitos entre otros. Los 4 proyectos financiados en Sincelejo lograron una inversión por parte del fondo emprender de \$323.502.400.oo, lo que significó el 40,61\% de la inversión lograda en el departamento y el $2,42 \%$ de lo invertido a nivel nacional.

Gráfico No. 10. Proyectos viables de la ciudad de Sincelejo, por entidad de apoyo

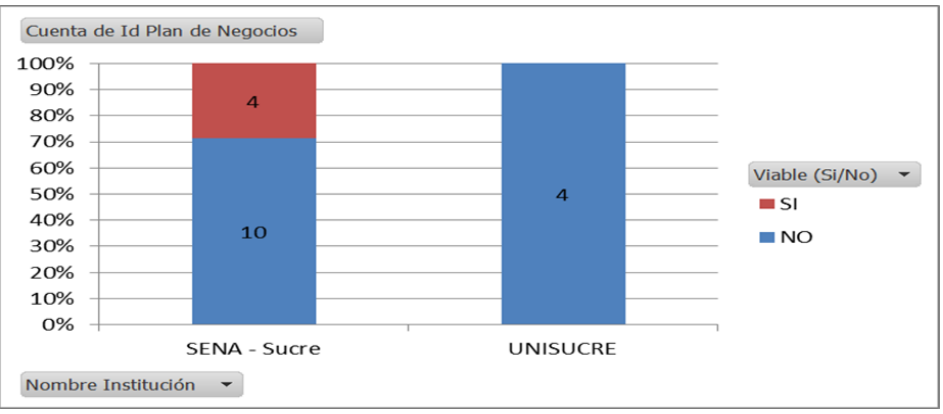

Para analizar la participación de las diferentes unidades de emprendimiento que operan en la ciudad de Sincelejo, Vale la pena mencionar que de los 42 proyectos presentados por emprendedores del departamento de Sucre, 18 fueron presentados por emprendedores de la ciudad de Sincelejo, de los cuales, 14 fueron presentados con el acompañamiento de la unidad de emprendimiento del Sena seccional Sucre y 4 fueron presentados con el acompañamiento de la unidad de emprendimiento de la Universidad de Sucre, los dos grandes ausentes de las convocatorias del fondo emprender 2011, en lo que a unidades de emprendimiento de la ciudad de Sincelejo se refiere, fueron la Corporación Incubadora de Empresas de Sucre y la unidad de Emprendimiento de la corporación Educativa CECAR (ver gráfico No 10).

De los 14 proyectos acompañados por la unidad de emprendimiento del Sena seccional Sucre, salieron los 4 proyectos viabilizados para la ciudad de Sincelejo, los 4 adicionales incubados por la unidad de emprendimiento de la Universidad de Sucre, todos fueron declarados no viables (ver gráfico No. 7).

Gráfico No. 11. Empresas Creadas En Sincelejo Por Subsector Convocatorias 2011

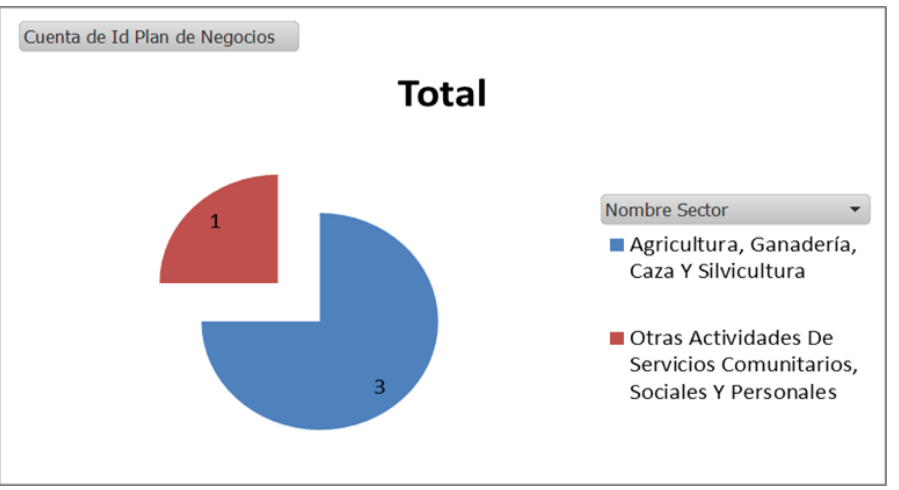

Los proyectos empresariales que lograron financiación del fondo emprender en la ciudad de Sincelejo, se ubicaron en los sectores de Agricultura, Ganadería, Caza y Silvicultura, con tres proyectos de los 4 financiados (ver gráfico No. 11). 


\section{CONCLUSIONES}

$7 \mathrm{n}$ la ciudad de Sincelejo se crearon, con la ayu$\triangle$ da de las entidades de apoyo, identificadas como unidades de emprendimiento y financiadas por el fondo emprender, 8 empresas en el año 2010 y 4 en el año 2011.

El nivel de inversión canalizado por los emprendedores en la ciudad de Sincelejo, con la orientación de las unidades de emprendimiento que operan en la ciudad y financiado por el fondo emprender, fue de $\$ 524.785 .000$ para el año 2010 y de $\$ 323.502 .400$ para el año 2011. Esa inversión representó el 2,2\% de lo invertido a nivel nacional y el 39,2 a nivel departamental para el año 2010 y el $2,42 \%$ a nivel nacional y 40,61 a nivel departamental, para el año 2011.

El sector de negocios más representativo, donde se crearon las empresas, producto de la financiación del fondo emprender y asesorados por la unidades de emprendimiento en la ciudad de Sincelejo, fue el sector caracterizado como "Agricultura, ganadería, caza y silvicultura".

La incidencia de las unidades de emprendimiento y el fondo emprender en la creación de empresas en la ciudad de Sincelejo, medida como el nivel de empresas creadas por efecto de dichas entidades y comparadas con el total de empresas creadas en la ciudad, para los años 2010 y 2011, fue de $8 \mathrm{em}-$ presas de 1227 y 4 empresas en 962 respectivamente. En términos relativos lo anterior se cuantifica como el $0,65 \%$ para el 2010 y el $0,41 \%$ para el 2011.

\section{Resultados adicionales que evidencia el proyecto}

$V_{\mathrm{cos}}^{\mathrm{a}}$ ale la pena resaltar algunos resultados que se evidencian con el presente proyecto y que no corresponden de una manera directa con los objetivos, pero que por su relevancia para el estema objeto de estudio podrían servir como puntos de referencia a futuras investigaciones.

Para los años 2010 y 2011, el departamento de Sucre ocupó los puestos 5 y 6 entre los departamentos donde los emprendedores canalizaron más recursos de inversión producto del fondo emprender. En el mismo sentido se evidencia, que los departamentos más involucrados en este tema son los departamentos de Huila, Nariño, Santander y el Distrito Capital, los cuales se ubicaron en los cinco primeros lugares de los departamentos que canalizaron mayor inversión, del fondo emprender, apropiándose del $35,4 \%$ y $38,25 \%$ de los recursos asignados para cada año respectivamente.

Los resultados reflejan, además, que la unidad de emprendimiento del Sena seccional Sucre, para el año 2011, fue la unidad de emprendimiento que incubó los proyectos financiados por el fondo emprender. En el mismo sentido se evidencia que los proyectos incubados en la unidad de la Universidad de Sucre, fueron declarados no viables y se nota ausencia total de las unidades de emprendimiento de la Corporación Educativa del Caribe CECAR y de la Corporación Incubadora de Empresas de $\mathrm{Su}-$ cre. 


\section{BIBLIOGRAFÍA}

Aedo, C. (2005). Evaluación del impacto. Santiago de Chile,: Naciones Unidas, CEPAL, CGZ .

Alburquerque Francisco, D. M. (2008). Guía de Aprendizaje Sobre Integración Productiva y Desarrollo Económico Territotial. El Efoque del Desarrollo Económico Territorial. (E. P. Isabel Barrera, Ed.) Sevilla, España: Instituto de Desarrollo Regional, Fundación Universitaria. Universidad de Sevi1la, Instituto de Economía, Geografía y Demografía, Consejo Superior de Investigaciones Científicas.

Alburquerque, F. (Abril de 2004). Desarrollo económico local y descentralización en América Latina. R E V I S TA D E L A C E PA L 8 2, 157-171. Alcaldía de Sincelejo. (2012). PLAN DE DESARROLLO MUNICIPAL 2012-2015 "UN ALTO COMPROMISO". SINCELEJO: ALCALDIA DE SINCELEJO.

Baker, J. L. (2000). Evaluación del impacto de los proyectos de desarrollo en la pobreza - Manual para profesionales. Washington D.C.: Banco Mundial.

Caicedo, C. J. (2008). Políticas e instituciones para el desarrollo económico territorial en América Latina y el Caribe. El caso de Colombia. Instituto Latinoamericano y del Caribe de Planificación Económica y Social (ILPES) . Santiago de CHile: Naciones Unidas -CEPAL.

Cala, H. A. (2005). Situación y necesidades de la pequeña y mediana empresa. (U. S. Arboleda, Ed.) Civilizar, 1-22.

CONFECAMARAS. (11 de Junio de 2011). 1.000 Empresa más grandes de Colombia. Poder(Edición 06-10), 28-128.

Congreso de Colombia. (10 de Julio de 2000). Ley 590. Bogota D.C.

Departamento Nacional de Planeación. (2008). Evaluación de Impacto del Fondo Colombiano de Modernización y Desarrollo Tecnológico de las Micro, Pequeñas y Medianas Empresas FOMIPYME. Bogotá D.C.: Sinergia - DNP.

Departamento Nacional de Planeación. (2010).
Evaluaciones en Competitividad. Bogotá: Sinergia. Gómez, L. (2004). Medidas de Apoyo a la Creación de Empresas en el Caribe Colombiano: análisis de la oferta y demanda de servicios. Barranquilla.

Ley, M. (2 de agosto de 2004). Ley 905 de 2004. Bogotá D C, Colombia.

Ministerio de Comercio Industria Turismo. (s.f.). Recuperado el 10 de Mayo de 2011, de www.mincomercio.gov.co

Ministerio de Comercio, I. Y. (2010). Perfil Departamento de Sucre. Bogotá D.C.: Oficina de Estudios Económicos.

Monsalves, M. (2002). Las PYME y los sistemas de apoyo en la innovación tecnológica en Chile. Santiago de Chile: Publicación de las Naciones Unidas. Murcia, C. H. (2011). Creatividad e Innovación Para el Desarrollo Empresarial. Bogotá: Ediciones de la U.

Pájaro, G. ,. (2007). Impacto de las Políticas de Apoyo a la Creación de Empresas en Cartagena de Indias Colombia, 2000-2005. Cartagena.

Pallares, F. (1988). Las Políticas Públicas El Sistema Político en Acción. Revista de Estudios Políticos (Nueva Epoca).

Parquesoft. (16 de 7 de 2012). Parquesoft Sucre. Recuperado el 16 de 7 de 2012, de http:// www.parquesoftsucre.com.idex.php $=$ com_content $\&$ view $=$ article $\&$ id $=91 \&$ Itemid $=206$

Tapia, G. J. (10 de 10 de 1993). Incidencia: Conceptos, terminología y análisis dimensional. Artículo Especial. Washintong D.C., Estados Unidos: Programa de publicaciones. Organización panamericana de la salud.

Varela, V. R. (2008). Innovación Empresarial "Arte y Ciencia en la Creación de Empresas. Santiego de Cali: Pearson, Prentice Hall.

Viceministerio de Desarrollo Empresarial. (08 de 08 de 2012). Portal- Ministerio de Comercio, Industria Y turismo. Recuperado el 18 de 09 de 2012, de https://www.mincomercio.gov.co/minindustria/ publicaciones.php?id=10171 